INPLASY

PROTOCOL

To cite: Li et al. Effect of inspiratory muscle training on ICU patients with mechanical ventilation:A systematic review and meta-analysis. Inplasy protocol 202170034. doi: 10.37766/inplasy2021.7.0034

Received: 11 July 2021

Published: 11 July 2021

Corresponding author: Ying Li

1784570921@qq.com

Author Affiliation:

Nanchang University. Jiangxi Medical College, Bayi Avenue, Donghu District, Nanchang City, Jiangxi Province.

Support: No.

Review Stage at time of this submission: Data analysis.

Conflicts of interest:

None declared.

\section{Effect of inspiratory muscle training on ICU patients with mechanical ventilation: A systematic review and meta-analysis}

\author{
Li, Y1; Zhou, J2; Fu, Y3; Li, D4.
}

Review question / Objective: Mechanical ventilation is an important means of respiratory support for critically ill patients. The survey shows that about $73 \%$ of ICU patients in China receive mechanical ventilation. IMT technology includes threshold load, resistance load and whole body mobilization. IMT has been proved to improve chronic obstructive pulmonary disease (COPD), stroke pulmonary function, chronic heart failure, and inspiratory muscle strength and exercise performance of athletes. At present, the rehabilitation work in intensive care unit mainly focuses on peripheral muscle dysfunction, while the training of respiratory muscle is relatively less concerned. In China, there are few researches and related reports on inspiratory muscle training of patients with mechanical ventilation. Objective To analyze the effect of inspiratory muscle training on respiratory muscle function in ICU patients with mechanical ventilation by meta analysis.

INPLASY registration number: This protocol was registered with the International Platform of Registered Systematic Review and Meta-Analysis Protocols (INPLASY) on 11 July 2021 and was last updated on 11 July 2021 (registration number INPLASY202170034).

\section{INTRODUCTION}

Review question / Objective: Mechanical ventilation is an important means of respiratory support for critically ill patients. The survey shows that about $73 \%$ of ICU patients in China receive mechanical ventilation. IMT technology includes threshold load, resistance load and whole body mobilization. IMT has been proved to improve chronic obstructive pulmonary disease (COPD), stroke pulmonary function, chronic heart failure, and inspiratory muscle strength and exercise performance of athletes. At present, the rehabilitation work in intensive care unit 
mainly focuses on peripheral muscle dysfunction, while the training of respiratory muscle is relatively less concerned. In China, there are few researches and related reports on inspiratory muscle training of patients with mechanical ventilation. Objective To analyze the effect of inspiratory muscle training on respiratory muscle function in ICU patients with mechanical ventilation by meta analysis.

Condition being studied: Mechanical ventilation is an important means of respiratory support for critically ill patients. The survey shows that about $73 \%$ of ICU patients in China receive mechanical ventilation. Mechanical ventilation is an important means to improve airway ventilation and oxygenation. During mechanical ventilation, because the patient's breathing is completely or partially provided by the ventilator, the diaphragm is in a completely unloaded state, and the contraction movement is significantly reduced, which leads to the disuse and atrophy of the diaphragm. Studies have shown that the incidence of disuse atrophy of diaphragm is twice that of other muscle atrophy. Once the diaphragm disuse atrophy can lead to ventilator dependence, difficult extubation, prolonged mechanical ventilation time, and increase the risk of ventilator associated pneumonia (VAP) and death.Inspiratory muscle training (IMT) aims to improve the strength and endurance of respiratory muscles by training diaphragm and auxiliary inspiratory muscles. IMT technology includes threshold load, resistance load and whole body mobilization. IMT has been proved to improve chronic obstructive pulmonary disease (COPD), stroke pulmonary function, chronic heart failure, and inspiratory muscle strength and exercise performance of athletes. At present, the rehabilitation work in intensive care unit mainly focuses on peripheral muscle dysfunction, while the training of respiratory muscle is relatively less concerned. In China, there are few researches and related reports on inspiratory muscle training of patients with mechanical ventilation. This study searched the literatures on inspiratory muscle training of patients with mechanical ventilation at home and abroad to explore the effect of inspiratory muscle training on respiratory muscle function of patients with mechanical ventilation in ICU, so as to provide basis for the rehabilitation of respiratory muscle function of patients with mechanical ventilation in ICU.

\section{METHODS}

Participant or population: ICU patients with mechanical ventilation.

Intervention: IMT was used in the experimental group.

Comparator: The control group received routine nursing or false IMT. The intervention time and frequency are not limited.

Study designs to be included: The included studies will be RCTS in this systematic review regardless of publication status and language.

Eligibility criteria: The PICOS principles were given full consideration to establish inclusion and exclusion criteria of this systematic review.

Information sources: Studies will be obtained from the China National Knowledge Infrastructure (CNKI), Wan Fang Data, Chinese Scientific Journals Database (VIP), PubMed, CBM, Embase, Web of science and Cochrane Library, regardless of publication date or language. The databases will be retrieved by combining the subject words with random words. Taking PubMed as an example, the retrieval strategy is shown in Table 1. The search terms will be adapted appropriately to conform to the different syntax rules of the different databases.

Main outcome(s): Main outcome measures: maximum inspiratory pressure after respiratory muscle training (Maxi mum inspiratory pressure,MIP). Secondary outcome measures: duration of mechanical 
ventilation; Oxygen partial pressure; Offline time.

Quality assessment / Risk of bias analysis: Two researchers (Yajun Fu and Jixin Zhou ) assessed the quality of the included RCTs independently by utilizing the Cochrane risk of bias assessment tool. As specified by Cochrane Handbook V.5.1.0 Bias Risk Assessment Manual to evaluate the quality of the included literature, and determine the high (A), medium (B), and low (C) grades of the literature based on the score[15]. The main evaluation contents include: random sequence generation, blind method, allocation concealment (to the research object, the investigator and the result assessor), completeness of the result data, selective reporting of research results and other sources of bias.

Strategy of data synthesis: Statistical software stata14.0 was used for metaanalysis. The outcome measure was the probability of time effect of counting data, and its $95 \%$ confidence interval $(\mathrm{Cl})$ was the effect quantity; The mean difference (MD) and its $95 \%$ confidence interval (CI) were used to represent the effect of measurement data. If there was no statistical heterogeneity among the studies ( $P>0.05,12<50 \%$ ), fixed effect model was used for analysis; On the contrary, random effect model is used for analysis. If there is statistical heterogeneity among the studies, analyze the source of heterogeneity and analyze the factors of possible heterogeneity.

Subgroup analysis: If there is high heterogeneity in the included studies, we will perform subgroup analyses to explore the differences in age, sex, race, lesion location, and course of the Intervention time.

Sensitivity analysis: To ensure robustness of the combined results, sensitivity analyses will be performed to assess the impact of studies with a high risk of bias.We will compare the results to determine whether lower-quality studies should be excluded.
Country(ies) involved: China.

Keywords: Inspiratory muscle training; Mechanical ventilation; Evidence based nursing.

Contributions of each author:

Author 1 - Ying Li.

Email: 1784570921@qq.com

Author 2 - Jixin Zhou.

Email: 1453457616@qq.com

Author 3 - Yajun Fu.

Email:651297951@qq.com

Author 4 - Dongying Li.

Email: a15775959359@163.com 